\title{
Influence of Laser Excitation on Raman and Photoluminescence Spectra and FTIR Study of Porous Silicon Layers
}

\author{
Walter Jaimes Salcedo ${ }^{1}$, Francisco J. Ramirez Fernandez ${ }^{2}$, and Joel C. Rubimc ${ }^{3}$ \\ ${ }^{1}$ Laboratório de Espectroscopia Molecular do Instituto de Química da USP \\ Av. Prof. Lineu Prestes, 748, 05508-900, São Paulo, SP, Brazil \\ ${ }^{2}$ Laboratório de Microeletrônica, Escola Politécnica, \\ Universidade de São Paulo, C.P.61548, 05424-970, São Paulo, SP, Brazil \\ 3 Universidade de Brasilia Departamento de Química \\ C.P. 04478, Campus Universitário Asa Norte 70919-970, Brasília-DF
}

Received February, 1998

\begin{abstract}
A porous silicon film (PS) was investigated by FTIR, Raman and photoluminescence (PL) spectroscopies. The Raman and PL spectra were obtained using four different laser excitations: 488, 514, 633 and $782 \mathrm{~nm}$. The analysis of the first order and second order Raman scattering lines permits to identify the band energy structure of the crystallites inside the PS film. The analysis of PL spectra shows that the intensity and full width at half-maximum values of PL emission depends on intensity and energy of laser excitation. The linear polarization degree (LPD) of the PL spectra also presents a dependence of laser excitation. The observed dependence of Raman and PL spectra due to laser excitation energy cannot be explained within the quantum confinement alone. We propose a mechanism for PL emission in PS layers, in which the radiative recombination occurs in localized centers at pore/crystallite interface. These quasi-molecular centers are Jahn-Teller active.
\end{abstract}

\section{Introduction}

Since the original reports of efficient photoluminescence (PL) from porous silicon (PS) $[1,2]$ the mechanism of the PL from PS is the subject of numerous investigations and discussions [3]. Although experimental evidence has been given for the quantum confinement model [4], ample arguments and experimental facts have shown that, in silicon nanocrystals with surface passivated by oxide, the PL originates from some radiative centers at the $n c-\mathrm{Si}_{/} \mathrm{SiO}_{2}$ interface [5]. One of the apparently unresolved problems is the large discrepancy between the calculated bad gap of the small silicon nanocrystals [6] and the observed distribution of PL spectra [7]. Here we discuss the surface structure, bonding and disorder in PS films related to PL emission using Fourier Transform Infra Red (FTIR), Raman and PL spectroscopies.

\section{Experiments and Results}

Porous silicon (PS) was prepared by electrochemical anodization using a $S i-p(100)$ oriented wafer with a resistivity of $10-20 \Omega \mathrm{cm}$. Before anodization, the back side of wafer was doped with Boron. Ohmic contact was made by evaporating an aluminum film. Three samples labeled as A, B and C were prepared. Sample A was prepared by anodization in $H F(49 \%): H_{2} O(1: 2)$, sample B in $H F(49 \%)$ : Ethanol(1:1) and sample $\mathrm{C}$ in $H F(49 \%)$. Anodization times were of 5, 10 and $30 \mathrm{~min}$ with current density of 3,25 and $40 \mathrm{~mA} / \mathrm{cm}^{2}$, respectively. The thickness of sample $\mathrm{A}, \mathrm{B}$ and $\mathrm{C}$ were of $5 \mu \mathrm{m}, 5 \mu \mathrm{m}$ and $30 \mu \mathrm{m}$ respectively.

The FTIR spectra of samples were obtained in the BIO-RAD system using transmission technique. The Raman and photoluminescence (PL) spectra were obtained on a Renishaw Raman system 3000 equipped with CCD detector and an Olympus BH-2 microscope. Measurements were made in the back scattering geometry using a $80 \times$ microscope objective to focus the laser beam on the sample. The area of the laser spot on the sample using the $80 \times$ objective is ca. $2 \mu m \times 2 \mu m$

In order to correlate surface structure of PS with PL emission, samples A and B were analyzed by FTIR spectroscopy (see Fig. 1). The PL spectra of this samples were obtained by excitation with The PL spectra of these samples were obtained by excitation with $488 \mathrm{~nm}$ laser source of $10 \mu \mathrm{Watts}$. 


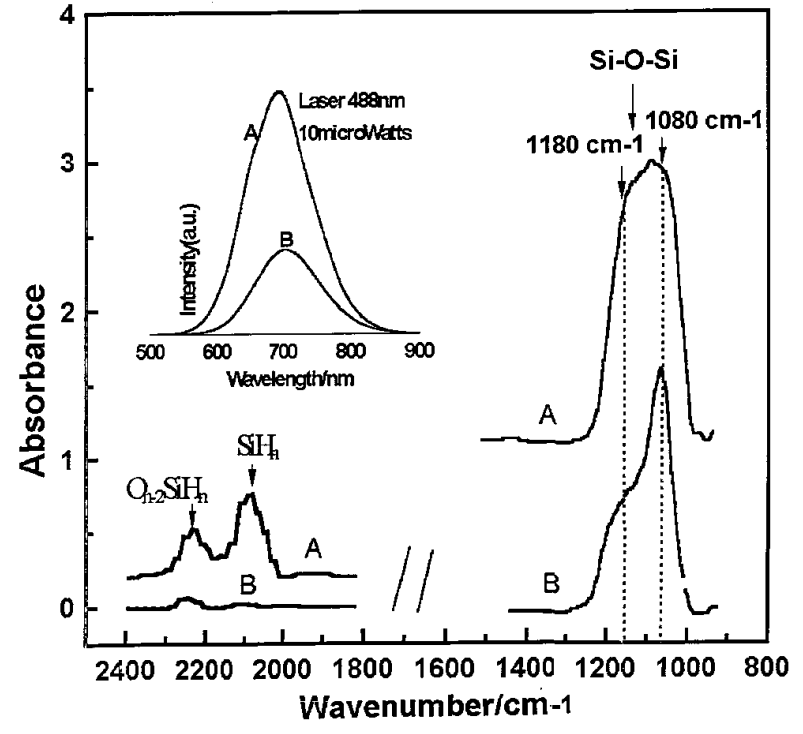

Figure 1. FTIR and PL spectra in sample A and B.

In order to investigate the effect of the laser power density in the PS structure, Raman and PL spectra were obtained before and after exposition of the samples to the maximum laser power $(15 \mathrm{~mW}$ at $514.5 \mathrm{~nm})$. To avoid laser damage of the sample before the samples are exposed to the high laser power density, the laser power was set to $50 \mu \mathrm{W}$. Sample $\mathrm{C}$ was cleaved and three micro regions labeled as I, II, and III were analyzed. Region I corresponds to Si/PS interface, Region II to the center of the PS layer, and Region III to the PS/Air interface (see the inset of Fig. 3). The Raman and PL spectra are shown in Figs. 2 and 3 respectively.

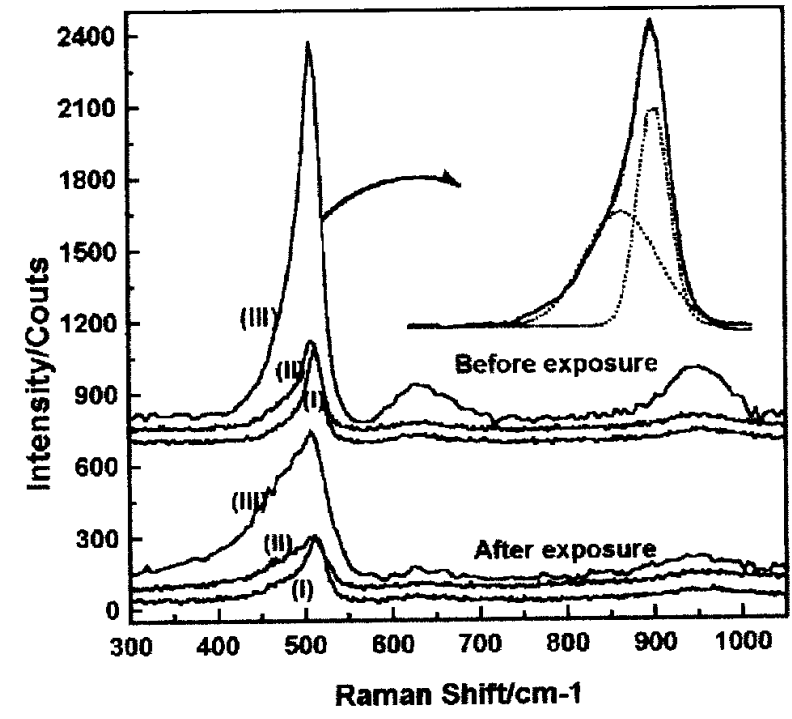

Figure 2. Effect of laser power on the Raman spectra of sample $\mathrm{C}$ (cleaved). The spectra were acquired before and after exposure of the different regions of the PS layer to $15 \mathrm{~mW}$ laser power (at $514.5 \mathrm{~nm}$ ). The laser power during spectral acquisition was $50 \mu W$. The inset shows the curve fitting in the $400-600 \mathrm{~cm}^{-1}$ spectral region. Regions I, II and III can be seen in the inset of Fig. 3 .

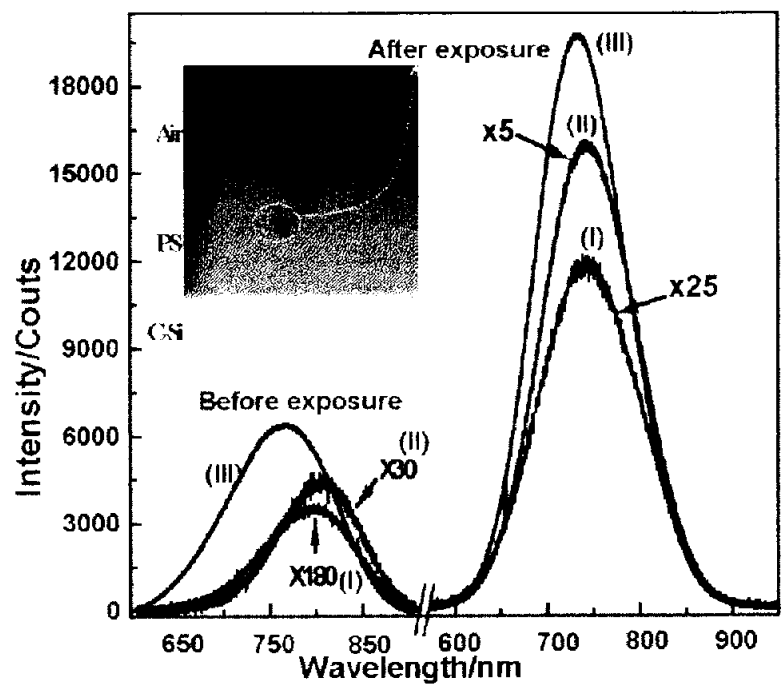

Figure 3. Effect of laser power on the PL spectra of sample $\mathrm{C}$ (cleaved). The spectra were acquired before and after exposure of the different regions of the PS layer to $15 \mathrm{~mW}$ laser power (at $514.5 \mathrm{~nm}$ ). The laser power during spectral acquisition was $50 \mu \mathrm{W}$. The inset shows the white light image of sample $\mathrm{C}$ indicating the relative position of Regions I, II and III in the sample. The image was obtained with the $\times 80$ objective.

Raman and PL spectra were recorded at $488 \mathrm{~nm}$, $514 \mathrm{~nm}$ (from an $\mathrm{Ar}^{+}$laser), $633 \mathrm{~nm}$ (from a $\mathrm{HeNe}$ laser), and $782 \mathrm{~nm}$ (from $\mathrm{Al} / \mathrm{GaAs}$ laser) excitations in order to study the dependence of the phonon and PL spectra on the laser excitation energy . The region II of cleaved sample $C$ was analyzed. The Figs. 4 and 5 show the Raman and PL spectra.

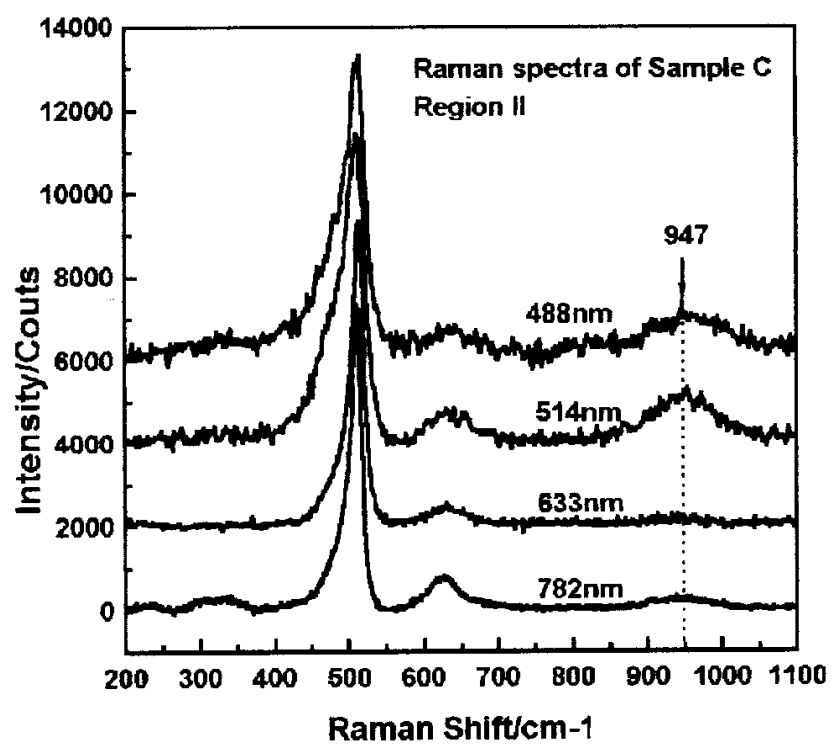

Figure 4. Raman spectra of sample $\mathrm{C}$ (cleaved) at region II. The spectra were excited with the $488.0,514.5,632.8$, and $782.0 \mathrm{~nm}$ laser radiations. 


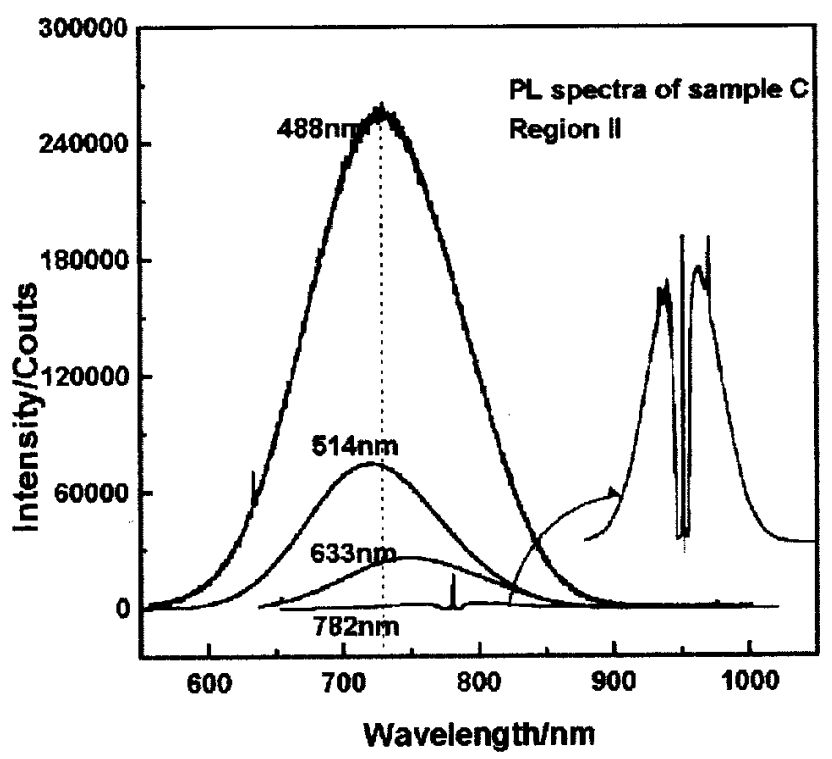

Figure 5. PL spectra of sample $\mathrm{C}$ (cleaved) at region II. The spectra were excited with the $488.0,514.5,632.8$, and $782.0 \mathrm{~nm}$ laser radiations.

Polarization measurements of PL spectra were performed using an analyzer after the notch filters and a quarter wave plate was set between the analyzer and the entrance slit of the monochromator to correct the grating response for different polarization of the scattered light. This experimental set-up for polarization measurements was tested with $\mathrm{CCl}_{4}$.

\section{Discussion}

\section{III.1 FTIR spectra}

The PS films A and B were prepared under different $H F$ concentrations. The FTIR absorption spectra of these samples are quite different in the $\nu\left(S i-H_{n}\right)$ stretching $\left(2100\right.$ to $\left.2300 \mathrm{~cm}^{-1}\right)$ and $\nu(S i-O-S i)$ stretching (1000 to $1250 \mathrm{~cm}^{-1}$ ) regions (See Fig. 1). Sample A (lower $H F$ concentration) shows stronger absorptions in these regions. Note also that the $\nu\left(S i-H_{n}\right)$ is stronger or sample $\mathrm{A}$. It is worth to mention that the stronger these absorption are, the stronger is the PL emission (See the inset of Fig. 1). Stutzman et. al. [8] have assigned the absorption features at 1080 and $1180 \mathrm{~cm}^{-1}$ to propose that this feature is due to $\nu(S i-O-S i)$ stretching modes. according to these authors, the surface of the Si-nanocrystallites by $S i_{6}$ regular rings containing $S i-O-S i$ bridges, forming a structure similar to the siloxene. Furthermore, they believe that these structures would be responsible for the PL emission on PS films. Following the Stutzman et. al. hypothesis, the enhancement of he PL emission in PS would be due to the increase of $S i_{6}$ structures. Therefore, the higher should be the absorptions in the 1000 to $1200 \mathrm{~cm}^{-1}$ region and their relative intensities should be remain constant. However, as one observes from Fig. 1, the relative intensity of the absorptions in this spectral region do not remain constant from sample A to B. In sample A (higher intensity for the PL emission) the absorptions at 1080 and $1180 \mathrm{~cm}^{-1}$ have almost the same intensity while for sample B the intensity of the feature at $1180 \mathrm{~cm}^{-1}$ is almost the half $\mathrm{f}$ that at $1080 \mathrm{~cm}^{-1}$. Deák et. al. [9] have theoretically estimated vibrational frequencies for different $S i-O-S i$ structures in silicon nanocrystallites. They have obtained a frequency value of $1079 \mathrm{~cm}^{-1}$ for the $S i-O-S i$ structure with vacancies that can be correlated to the absorption feature at $1080 \mathrm{~cm}^{-1}$ in the PS layer. The feature at $1180 \mathrm{~cm}^{-1}$ can be assigned to $n u(S i-O S i$ in the $S i-O-S i$ structure without vacancies in agreement with the observations in th ref [10]. The upper shift of this absorption band is due to the electronegative of the neighborhood [10]. According to our results, th feature at $1180 \mathrm{~cm}^{-1}$ is well and directly correlated to the PL emission, contrary to the hypothesis of Lowe et. al. that proposed the $\mathrm{Si}-\mathrm{O}-\mathrm{Si}$ structure with vacancies $\left(\nu(S i-O-S i)=1080 \mathrm{~cm}^{-1}\right.$, called "not bridge oxygen hole center" (NBOHC), as the surface structure responsible for $\mathrm{PL}$ emission.

\section{2 Raman Spectra}

The LO phonon Raman peak, in the PS film of sample $\mathrm{C}$ obtained with the $514 \mathrm{~nm}$ laser source, shows a downshift $\left(510 \mathrm{~cm}^{-1}-512 \mathrm{~cm}^{-1}\right)$ relative to LO Raman peak of $c-S i\left(520 \mathrm{~cm}^{-1}\right)$. Using the phonon confinement model [12], it was determined that crystallites size in PS film are in the range of $2.8 \mathrm{~nm}$ to $3.2 \mathrm{~nm}$. The best fit was obtained considering a spherical shape to crystallites. In addition, it was observed another band in the region of $480 \mathrm{~cm}^{-1}$ that was obtained by decomposition of band in the region of $400 \mathrm{~cm}^{-1}$ to $550 \mathrm{~cm}^{-1}$ (see Fig. 2). Stuzman et. al. [8] attributed this band to $S i_{6}$ structure, this structure have $D_{3} d$ symmetry, so the band should be polarized. However, in previous work Jaimes et. al. [13] showed that this peak presents depolarised feature. The $480 \mathrm{~cm}^{-1}$ band is attributed to interface region structure formed by $S i-O-S i R$ or $S i-S i R$ where $R$ can be $H_{n}$ or $O H$ or other radicals. When the PS structure was exposed to the maximum power of the laser source the region of area exposed showed a dark brown color feature. At this region, the peaks of LO phonon Raman did not change the structure, i.e., the crystallite size calculated before is conserved. However, the peak at $480 \mathrm{~cm}^{-1}$ showed an increase of intensity and full width at half maximum (FWHM) of the band relative to LO phonon Raman band. This feature is correlated with the PL emission intensity increasing as Fig. 3 shows. With the FTIR technique it was observed that $S i-O-S i$ structure without vacancies is correlated with higher PL intensity. On the other side, using the LO phonon Raman peak and FWHM in silicon crystal the temperature at 
the surface area of the spot in the sample was estimated. The value was approximately $600{ }^{\circ} \mathrm{C}$ when the laser source was of high intensity.

It was reported that $S i-H_{n}$ bonds are broken at temperatures above $500{ }^{\circ} \mathrm{C}$ [14]. In the present case the experiment was made at room temperature under a relative humidity of $60 \%$. During laser exposure, $H$ desorption is produced from the PS surface. After that the $\mathrm{H}_{2} \mathrm{O}$ molecule reacts with the PS surface forming the $\mathrm{Si}-\mathrm{O}-\mathrm{SiH}_{n}$ or $\mathrm{Si}-\mathrm{O}-\mathrm{SiOH}$ as reported by [15], so these structures are directly responsible for the $480 \mathrm{~cm}^{-1}$ peak in the Raman spectra.

The second order Raman peak at $630 \mathrm{~cm}^{-1}$ and $960 \mathrm{~cm}^{-1}$ did not show changes in the intensity relative to LO phonon Raman peak showing that these peaks do not depend on the surface structure of PS indicating that these features correspond to $S i-S i$ vibration in the crystallites. The peak at $630 \mathrm{~cm}^{-1}$ is assigned to $\mathrm{TO}(\mathrm{X})+\mathrm{TA}(\mathrm{X})$ contribution and the peak at $960 \mathrm{~cm}^{-1}$ is assigned to $2 \mathrm{TO}(\mathrm{X}), 2 \mathrm{TO}(\mathrm{W})$ and $2 \mathrm{TO}(\mathrm{L})$ contribution as it was suggested in [16]. On the other side, the change of position peaks of PL emission band (see Fig. 3) after laser source exposure $(15 \mathrm{~mW})$ can be explained as a result of changing $R$ radical in the structure of $S i-O-S i R$ (eg. $H$ by $O H$ ). J. L. Gole et. al. [17] calculated the band gap of the poliatomic molecules with structure of SiOSiR showing that changing the $R$ radical provoke a changing in the band gap of molecule.

Fig. 4 displays the phonon Raman spectra of sample $\mathrm{C}$ at four different laser excitations. Note that the LO Raman phonon (at ca. $510 \mathrm{~cm}^{-1}$ ) does not depend on the energy on the laser excitation, since the observed Raman peaks present no shift within the experimental error $\left( \pm 1 \mathrm{~cm}^{-1}\right)$. Delerue et. al. [6] have proposed that the radiative recombination is due to direct exciton recombination suggesting that there exists a direct dipole transition in the band structure of the crystallites. However, we were not able to say if there is any resonance for the PS LO Raman phonon since we do not have a internal standard. However, the data displayed in Fig. 4 clearly show that there is a significant change on the intensity of the second order Raman peak at $950 \mathrm{~cm}^{-1}$ relative to the LO Raman phonon of PS (at $510 \mathrm{~cm}^{-1}$ ). If we consider the intensity ratio $I_{950} / I_{510}$, it is larger at 514.5 and $488.0 \mathrm{~nm}$ excitation as compared to the 632.8 and $782 \mathrm{~nm}$ excitations. Using the calculated size of crystallites and the quantum confinement model [6], the band gap in the crystallites were estimated to be ca. 2.15 to $2.47 \mathrm{eV}$. The laser excitations at 488.0 and $514.5 \mathrm{~nm}$ correspond to 2.54 and $2.20 \mathrm{eV}$, respectively. Therefore, the enhancement of the $950 \mathrm{~cm}^{-1}$, relative to the LO phonon can be explained as an indirect band gap resonance as in $c-S i$ [18].

\section{III.3 Photoluminescence}

The intensity and maximum of the PL emission are dependent on the laser excitation energy (see Fig. 5). The higher is the laser energy the more intense are the PL emission. This is an indication that the light absorption process within the PS layer is dependent on the energy band structure of the crystallite as reported by Baierle et al. [19]. However, we were able to excite the PL emission with a laser energy $(782 \mathrm{~nm})$ that is very close to the PL maximum, showing that the PL emission of PS layers is not directly depend on the crystallite energy band structure as it was suggested for many researchers $[1,6,20]$.

It is worth to mention that the maximum of PL emission does also depend on the laser excitation energy. This observation can be explained by the heterogeneous surface chemistry presented by the $S i$ nanocrystallites. In other words, the majority of emitters at $488.0 \mathrm{~nm}$ excitation have a chemical composition that is different from the majority of emitters at $632.8 \mathrm{~nm}$ excitation. According to the above discussion, the PL emission is associated with the presence of $S i-O-S i-R$ structures on the surface of the nanocrystallites. Therefore, the changes on the PL emission maximum upon laser energy can be accounted by the changes in the chemical nature of $R$.

The linear polarization degree (LPD) is defined as $\left(I_{\|}-I_{\perp}\right) /\left(I_{\|}+I_{\perp}\right)$, where $I_{\|}$and $I_{\perp}$ represent the emission band intensity in parallel and perpendicular polarization respectively. Fig. 6 shows the spectra of the LPD coefficient. At $632.8 \mathrm{~nm}$ excitation, the LPD coefficient shows higher polarization degree at higher energies decreasing slowly for lower emission energies. On the other hand the LPD coefficient shows a dependence on the laser excitation. The LPD values are higher for lower laser energies. Comparing the wavelength of the lasers sources to the crystallite size $(2.3 \mathrm{~nm}$ to $3.2 \mathrm{~nm}$ ) one can consider that the electric field inside the nanocrystallites is homogeneous and the dependence of the LPD coefficient on the laser excitation can not be explained as a depolarization effect inside the crystallites.

From the above results based on FTIR, Raman, and PL measurements we can picture the idea that the radiative recombination of $e-h$ pairs occurs on localized centers at the $S i$ nanocrystallite/pore interface in the PS layer. We believe that the $S i-O-S i-R$ structures form these localized centers. Since these centers are formed by polyatomic molecules we believe that the radiative recombination is a phonon assisted as indicated by the Jahn - Teller theorem [21]. Within this model, the LPD behavior can be explained as follows: The laser source of higher energy excites the electrons from the ground to an excited state of higher energy. Due to electron phonon interaction the excited state has different minima. Before recombination, the electron can be pumped by a phonon to another minimum with different symmetry and different polarization. The further 
radiative recombination at this minimum produces the depolarization of the emission band.

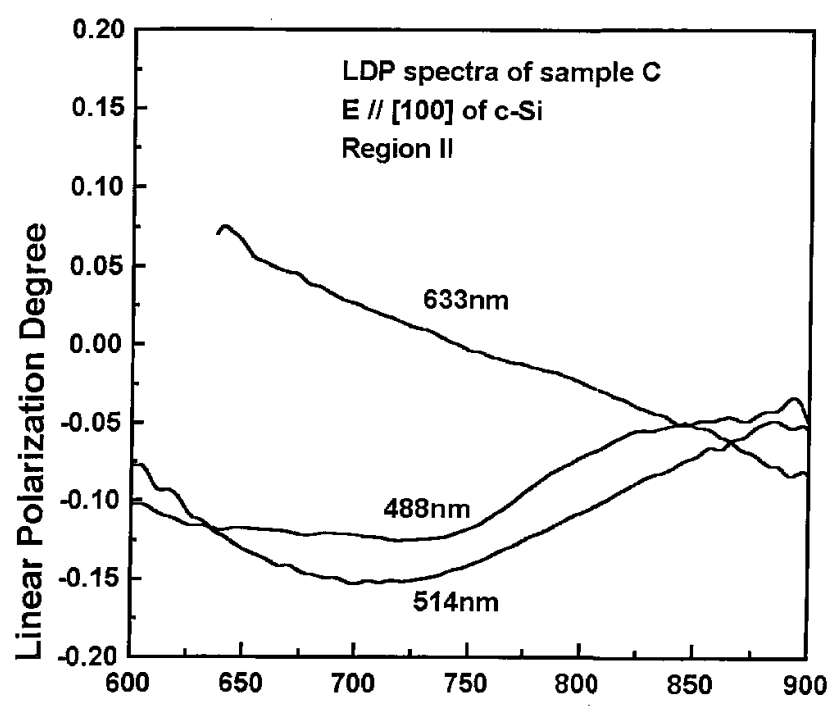

Figure 6. Linear Polarization Degree (LPD) spectra obtained by excitation of cleaved sample $\mathrm{C}$ at region II. The laser sources were of $488 \mathrm{~nm}, 514 \mathrm{~nm}$ and $633 \mathrm{~nm}$, respectively. The incident electric field was in the [100] direction of $c-S i$

\section{Conclusion}

Contrary to other infrared studies [8] the results presented here show that PL emission is correlated to $S i-O-S i R$ structures. These structures have originated localized centers as poliatomic structures. The LO phonon Raman spectra in PS does not show a resonant behavior. However the second order Raman line at $950 \mathrm{~cm}^{-1}$ presents an indirect band gap resonance as in $c-S i$. These results show that the energy band structure inside crystallites have an indirect band gap as in the $c-S i$. The Raman line at $480 \mathrm{~cm}^{-1}$ that is correlated to PL emission intensity is related to surface structure in the PS film $(S i-O S i R)$. The PL emission band intensity of PS layer presents a dependence on laser source excitation indicating the absorption dependence on band energy structure inside of crystallite. The LPD coefficient shows laser source dependence, indicating the localized state as responsible for the radiative recombination in PS film. The FTIR, Raman and PL spectra show that the localized centers are responsible for the radiative recombination in PS films. The localized centers are formed by poliatomic system that promotes electron-phonon interactions called Jahn-Teller effects.

\section{Acknowledgements}

We acknowledge FAPESP for financial support.

\section{References}

[1] L. T. Canham, Appl. Phys. Lett. 57, 1046 (1990).

[2] V. Lehmann and U. Gosele, Appl. Phys. Lett. (1991).

[3] M.S. Hybertsen, in: Porous Silicon Technology, eds. J. C. Vidal and J. Derrien, Springer-Verlag, Berlin 1995, p. 67 .

[4] P. D. J. Calcott, N. K. J. Nash, L.T. Canham, M. J. Kane, M. D. Brunhead, J. Phys. C 5, L91 (1993).

[5] F. Koch, V. Petrova-Koch and T. Muschik, J. Lumin. 57, 271 (1993).

[6] C. Delerue, M. Lannoo and G. Allan, J. Lumin, 57, 249 (1993).

[7] S. M. Prokes, Appl. Phys. 62, 3244 (1993).

[8] M. Stutzmann, Phys. Stat. Sol. (b), 192, 273 (1995).

[9] P. Deak, L. C. Snyder and J. W. Corbett; Phys. Rev. B, V. 45, 11612, 15 May 1992 - II.

[10] M. Nakamura, Y. Mochizuki, K. Usami; Sol. Stat. Commun., V. 50, 1079 (1984).

[11] R. R. Lowe-Webb, H. Lee, J. B. Ewing, S. R. Collins, W. Yang and P. C. Sercel; J. Appl. Phys., V. 83, 2815, 1 March 1998.

[12] N. Bruneto, G. Amato; Thin Solid Films, V. 297, 122 (1997).

[13] Walter J. Salcedo - Francisco J. R. Fernandez and Joel C. In edition J. Raman Spectroscopy 1999.

[14] H. D. Fuchs, M. Stutzman, M. S. Brandt, M. Rosenbauer, J. Weber, A. Breitschwerd, P. Deak and M. Cardona; Phys. Rev. B, V. 48, 8172, 15 September 1993-I.

[15] P. Gupta, Surf. Sci. V. 245, 360 (1991).

[16] H. Tanino, A. Kupring and H. Deai, Phys. Rev. B. 53, 1937 (1996).

[17] J. L. Gole, D. A. Dixon; J. Phys. Chem. B, V. 102, 33 (1998).

[18] P. B. Klein, H. Masui, J. Song, R. K. Chang; Solid state Commun, V. 14, 1163 (1974).

[19] R. J. Baierle, M. J. Caldas, E. Molinari and S. Ossicini; Sol. Stat. Commun., V. 102, 545 (1997).

[20] L. T. Canham; Mat. Res. Soc. Symp. Proc. V. 452, 29 (1997).

[21] Y. E. Perlin, M Wagner; N. H, Elsevier Science Publishers B. V., 1984. 